\title{
Les fondations de la Communauté de Sant'Egidio et de la Société de Saint-Vincent-de-Paul
}

Essai de mise en parallèle

\section{Charles Mercier}

\section{(2) OpenEdition}

\section{Journals}

Édition électronique

URL : http://journals.openedition.org/assr/22009

DOI : 10.4000/assr.22009

ISSN : 1777-5825

\section{Éditeur}

Éditions de l'EHESS

Édition imprimée

Date de publication : 31 mars 2010

ISBN : 978-2-7132-2253-5

ISSN : 0335-5985

\section{Référence électronique}

Charles Mercier, «Les fondations de la Communauté de Sant'Egidio et de la Société de Saint-Vincentde-Paul », Archives de sciences sociales des religions [En ligne], 149 | janvier-mars 2010, mis en ligne le 13 décembre 2010, consulté le 19 avril 2019. URL : http://journals.openedition.org/assr/22009 ; DOI 10.4000/assr.22009 


\section{Charles Mercier}

\section{Sant'Egidio et Saint-Vincent-de-Paul}

Dans un livre-entretien avec Dominique Chivot, paru récemment chez Bayard, Andrea Riccardi, historien et président de la communauté de Sant'Egidio, écrit à propos des débuts de ce groupe qu'il a fondé à Rome en février 1968 : «Nous nous disions : ne faisons pas les dames de Saint-Vincent-de-Paul; ne portons pas de cadeaux comme dans un rapport de dépendance entre bourgeois et pauvres; créons un lien d'amitié. Et nous avons fait une école d'alphabétisation dans ces baraquements » (Riccardi, 2001 : 21). Dans la décision pratique qui est à prendre par la communauté naissante de Sant'Egidio (comment vivre concrètement le service des pauvres ?) le modèle de la Société de Saint-Vincent-de-Paul apparaît comme un repoussoir. Pourtant, il existe des points communs remarquables entre cette dernière et la communauté de Sant'Egidio : il s'agit de deux organisations catholiques de laïcs, fondées par des jeunes gens aux profils similaires et ayant pour principal domaine d'action le service des pauvres. Aussi est-il tentant d'essayer de comparer plus profondément ces deux organisations.

Celles-ci ayant été fondées à cent trente-cinq ans d'intervalle ${ }^{1}$, leur mise en parallèle présente des difficultés. Sant'Egidio est une " communauté nouvelle » tandis que la Société de Saint-Vincent-de-Paul est une organisation déjà ancienne. Par conséquent l'étude ne portera pas sur les deux organisations actuelles mais sur leurs débuts respectifs et, plus particulièrement, sur la période de leur fondation. Celle-ci peut être définie comme le laps de temps durant lequel sont posées les bases de l'organisation. Elle est encadrée par une première réunion à caractère informel d'un côté et par la mise en place de statuts qui assurent la pérennité à la structure de l'autre. Pour la Société de Saint-Vincent-de-Paul, il s'agit d'une période relativement brève, deux ans et demi, allant d'avril 1833 (première réunion) à décembre 1835 (promulgation du règlement). Pour la communauté de Sant'Egidio, cette période est plus étirée : la rédaction des statuts n'intervient qu'en 1986, soit dix-huit ans après les débuts de la communauté en février 1968. À la lumière de cette comparaison, peut-on parler d'un modèle commun à la communauté de Sant'Egidio et à la Société de Saint-Vincent-de-Paul, ce qui induirait que les membres de Sant'Egidio « revivent » l'expérience d'Ozanam et de ses amis avec

1. La Conférence de charité qui donnera naissance à la Société de Saint-Vincent-de-Paul a, en effet, été fondée en 1833. 
un décalage de cent cinquante ans ? Est-on en présence, au contraire, de deux modèles distincts ?

Le travail de comparaison portera sur trois aspects : la construction de l'identité du groupe, les formes de communalisation et la durée de la phase de domination charismatique.

\section{La construction de l'identité religieuse du groupe}

L'identité religieuse entendue dans un sens large ne concerne pas simplement le contenu des croyances mais aussi les pratiques religieuses, les manières de concevoir le monde et d'y agir, les appartenances vécues (Hervieu-Léger, 2001 : 70). Elle se construit nécessairement par l'insertion dans une lignée croyante et le rattachement à une tradition (Hervieu-Léger, 1993). Comment s'opère, pour la Société de Saint-Vincent-de-Paul et la communauté de Sant'Egidio, ce rattachement à une ascendance de croyants, condition de la fabrication d'une identité religieuse ?

\section{Le thème de la rupture}

Les deux groupes à leur début se pensent en rupture avec le passé immédiat. Les fondateurs de la Société de Saint-Vincent-de-Paul veulent démarquer leur structure des œuvres de la Congrégation, une association religieuse fondée à Paris, en 1801, par un ancien jésuite, l'abbé Delpuits. À l'origine œuvre de piété et de charité qui ne rassemblait que quelques étudiants, marquée par le retour des Bourbons, elle regroupe à partir de l'année 1814 de plus en plus de personnages influents qui lui donnent un poids non négligeable sur le plan religieux, social, voire politique. Parallèlement à la création d'une "filiale » caritative (la Société des bonnes œuvres, spécialisée dans la visite des hôpitaux et des prisons), la Congrégation encourage certains de ses membres à se regrouper dans une association secrète, les chevaliers de la foi, qui se veut au service de l'alliance du Trône et de l'Autel (Bertier de Sauvigny, 1948). Ce mélange des genres, déjà dénoncé sous la Restauration (Montlosier, 1826), est fatal à la Congrégation qui ne survit pas à la Révolution de 1830. Les confrères de Saint-Vincent-de-Paul cherchent à se prémunir de ces errements : dans le règlement promulgué en 1835, ils décident d'évacuer l'action proprement politique de leur association : «L'esprit de charité en même temps que la prudence chrétienne nous portera encore à bannir à jamais de nos réunions communes ou particulières les discussions politiques (...). Notre société est toute de charité : la politique lui est tout à fait étrangère " (Société de Saint-Vincent-de-Paul, 1836 : 22-23). Par ailleurs, au secret, susceptible d'alimenter les rumeurs et les légendes, est préférée l'humilité ${ }^{2}$.

2. «Quoique nous aimions davantage notre petite association, nous l'estimerons toujours moins excellente que les autres; nous ne verrons en elle, comme elle l'est en effet, qu'une œuvre formée par on ne sait qui, ni comment, née d'hier et qui peut mourir demain. " (Société de Saint-Vincent-de-Paul, $1836: 19$ ). 
Pour ce qui concerne la communauté de Sant'Egidio, la phrase d'Andrea Riccardi citée en introduction témoigne d'une volonté de rupture par rapport à l'organisation catholique caritative devenue dominante : la Société de SaintVincent-de-Paul. Mais la volonté de démarcation est plus large. C'est l'institution catholique toute entière qui apparaît comme un repoussoir : "l'Église officielle ne nous plaisait pas trop, instinctivement, parce qu'elle était une institution un peu trop impliquée avec le pouvoir. Surtout elle était loin, tout en étant à Rome » (Riccardi, 1996 : 12). Le contexte de la seconde moitié des années soixante alimente cette volonté de changement radical : «J'ai lu Gramsci et un peu Marx en 1968. C'était une génération qui les découvrait. J'avais surtout l'idée que le monde devait changer, qu'il fallait s'interroger sur la manière de le changer, qu'il fallait inventer l'avenir, changer les règles du jeu, tracer les lignes de développement » (ibid. : 6).

Le fait que les deux groupes construisent leur identité religieuse en rupture avec ce qui précède immédiatement n'est pas en soi surprenant. La fondation d'une nouvelle organisation n'est légitime que dans la mesure où elle apporte du nouveau par rapport aux structures déjà existantes. De plus, dans les cas d'organisations de jeunesse, la nouvelle génération montante ressent souvent le besoin de se démarquer des aînés (Cholvy, 2000 : 356 ; Hervieu-Léger, 2001 : 62). La question est donc de savoir si, au-delà de la rupture identitaire imaginée par les fondateurs, il y a réellement une crise de la transmission de l'identité religieuse.

\section{Deux situations de crise de la transmission religieuse?}

Dans les deux cas, les fondations sont opérées dans des situations où le lien constitutif de la religion à la mémoire est distendu, semblant mettre en péril la continuité des générations croyantes.

La fondation de la Société de Saint-Vincent-de-Paul a lieu dans les années 1830, dans un contexte où la mémoire collective religieuse est fragilisée par cette grande rupture que constitue la Révolution. Au début de la décennie 1830, le clergé n'est pas encore tout à fait remis de la désorganisation religieuse provoquée par la Constitution civile du clergé, la création de deux Églises parallèles, les persécutions. Dans de nombreuses familles, l'épisode révolutionnaire a entraîné des ruptures dans la chaîne de croyance aux dogmes du catholicisme. Ainsi, parmi les sept fondateurs de la Société, on compte un étudiant saint-simonien récemment converti (Société de Saint-Vincent-de-Paul, 1960 : 16). Des pans entiers de la société ont oublié le passé religieux. À cette perte de mémoire s'ajoute, dans cette même période, la difficulté de la mobilisation imaginaire du passé pour l'invention de l'avenir. La réalité nouvelle issue de la Révolution de juillet semble trop complexe, trop différente de celle connue par les générations précédentes. Cet événement ne marque pas seulement un essai de révolution politique et sociale, c'est « un tournant dans l'évolution générale de la société qui semble 
"vaciller" sur des assises idéologiques traditionnelles remises en questions en elles-mêmes ou par l'éclosion et la diffusion de nouvelles technologies " (Brémard, 1967 : 15). Cette révolution marque pour les catholiques la fin du rêve d'alliance entre le Trône et l'Autel. Les émeutes sociales qui l'accompagnent font prendre conscience de l'avènement d'une société industrielle marquée par les conflits entre pauvres et riches.

La fondation de la communauté de Sant'Egidio a lieu en 1968. La mémoire collective religieuse est également menacée en cette période de développement économique et d'exode rural. Andrea Riccardi raconte que sa famille s'est détachée de la religion en quittant l'Ombrie pour Rome : "Ma famille, après que les nouvelles générations eurent quitté l'Ombrie, est devenue plutôt laïque. Mon père, président d'une banque appartenait à cette tradition. Il était lié à $\mathrm{Il}$ mondo de Pannunzio, cette revue laïque de la gauche non marxiste, de haut niveau culturel, qui dans l'Italie de l'après-guerre était un vrai modèle de journalisme. Le climat religieux était tolérant, ni religieux, ni laïciste, ni anticlérical. Ma mère aussi était plutôt laïque» (Riccardi, 1996 : 3). En ces années, la conjonction de la réception du concile Vatican II et de la montée de la contestation étudiante bouleverse considérablement les modalités de la transmission religieuse, posant la question de sa légitimité même. A. Riccardi note que le rôle de transmetteur du prêtre était en pleine recomposition : "J'ai vu de près la crise du prêtre après Vatican II : devait-il être un leader ou pas ? Il n'avait plus un rôle aussi sûr dans l'Église... » (ibid. : 12).

La crise de la transmission semble donc marquer le contexte de chaque fondation. Pourtant, il semble que ces deux crises sont d'une importance inégale.

\section{Deux crises d'une inégale importance}

L'identité religieuse de la Société de Saint-Vincent-de-Paul est façonnée en grande partie par les deux adultes vers qui se tournent les étudiants : Emmanuel Bailly, âgé de quarante-deux ans en 1833, éditeur, ancien membre de la Congrégation, et Rosalie Rendu, âgée de quarante-sept ans, sœur de la charité, s'occupant des pauvres de la rue Mouffetard.

Emmanuel Bailly, élu président de la Conférence de charité, qui donnera naissance à la Société de Saint-Vincent-de-Paul, rédige les "Considérations préliminaires du règlement » qui affilient les confrères à la spiritualité de Vincent de Paul. Pour l'auteur anonyme d'un article publié dans La Documentation Catholique, en 1926, ces considérations préliminaires ne font qu'entériner un fait dont les fondateurs n'étaient pas conscients : ils ont, sans s'en rendre compte, ressuscité les œuvres que Vincent de Paul avaient créées deux siècles auparavant : «Les conférences de charité n'étaient-elles pas, en somme, la résurrection, sous une forme nouvelle, de celles que saint Vincent de Paul avait établies en son temps, d'abord avec les femmes, par les Confréries de charité encore vivantes ; 
ensuite avec M. de Renty par l'Association des Gentilshommes, que les nouvelles Conférences faisaient en quelque sorte revivre ? On avait imité ces diverses organisations sans s'en rendre bien compte; c'était comme une conséquence, non aperçue tout d'abord, de la longue formation de M. Bailly à l'école de saint Vincent de Paul (...). À force d'en méditer la vie, les exemples et les écrits, il s'était pour ainsi dire assimilé les préoccupations, les désirs, et même le caractère et jusqu'au style du saint » (1926 : 598). Cette filiation avec Vincent de Paul apparaît pleinement dans le nom donné à l'organisation par le Règlement : Société de SaintVincent-de-Paul.

L'autre adulte référent qui transmet une identité religieuse dans le domaine des pratiques charitables est la sœur Rosalie Rendu. En 1833, cette dernière préside à la distribution des secours du bureau de bienfaisance du XII ${ }^{\mathrm{e}}$ arrondissement, qui correspond à l'actuel quartier de la rue Mouffetard (Sullivan, 2007 : 208). Elle a, à l'époque, une solide réputation de dévouement pour les pauvres puisqu'elle a fondé une crèche et un asile pour les miséreux et s'est dévouée sans compter pour les cholériques en 1832 (Goyau, 1926 : 584). Comme le raconteront des fondateurs survivants en 1882 , les étudiants ne connaissent pas personnellement de pauvres à secourir et ignorent les pratiques par lesquelles on peut leur venir en aide. Ils choisissent donc de s'adresser à cette spécialiste de la charité qu'est Rosalie Rendu. Celle-ci leur fournit des bons à remettre aux nécessiteux ainsi que des conseils pratiques (Société de Saint-Vincent-de-Paul, 1960 : 18). Ce transfert de savoirs, de compétences et d'attitudes de Rosalie Rendu vers les premiers confrères renforce la constitution de l'identité vincentienne de la conférence de charité. En effet, Rosalie Rendu fait partie de la communauté des filles de la charité fondée, en 1633, par Vincent de Paul et Louise de Marillac.

Plus ou moins consciemment, l'identité religieuse s'est construite par la médiation de deux adultes fortement imprégnés de spiritualité vincentienne. Le "bricolage identitaire " reste limité. Les éléments sont tous puisés à la même source : Vincent de Paul. En 1849, Frédéric Ozanam le considère comme le maître de l'organisation qu'il a contribué à fonder : «Et reportant maintenant nos regards vers notre maître saint Vincent de Paul, songeons que lui aussi était venu dans des temps difficiles... Son œuvre ne vieillit pas ; qui ne voudrait la recommencer ? " (Société de Saint-Vincent-de-Paul, 2003 : 11). Plus tard, les récits des origines mettront en valeur la complémentarité entre l'enracinement dans une tradition et l'ouverture à la nouveauté dans le processus de la fondation. L'analyse sémiotique d'un récit des origines composé en 1882 par trois fondateurs survivants ${ }^{3}$ montre que les figures liées au passé, incarné par Bailly, et les figures liées à la jeunesse, incarnée par Ozanam, cohabitent harmonieusement (Mercier, 2006 : 79-83).

3. François Lallier, Auguste Le Taillandier et Paul Lamache. Ce récit des origines est publié pour la première fois dans le Bulletin de la Société de Saint-Vincent-de-Paul en 1882. 
La constitution de l'identité de Sant'Egidio est beaucoup plus complexe. Elle s'opère en l'absence d'adultes référents. Andrea Riccardi, qui a fondé son groupe, ressent le besoin de se former religieusement. Il a recours aux livres dans une démarche totalement personnelle : "J'éprouvais le sentiment que si l'Évangile suffit, il n'en est pas moins nécessaire d'avoir un minimum de culture religieuse et de s'orienter. Je commençais à me doter d'une culture théologique. Je me suis donc mis à lire Congar, Chenu, de Lubac, Rahner, un peu de théologie postconciliaire (...). Je me donnais une formation théologique personnelle mais je n'avais aucun contact avec l'Église officielle» (1996: 10-11). A. Riccardi procède donc à une auto-construction de sa culture religieuse. Il est en quelque sorte un "autodidacte de la foi » (ibid.: 12). La démarche est bien différente de celle effectuée par les premiers confrères de la Société de Saint-Vincent-de-Paul et illustre le propos de Danièle Hervieu-Léger concernant la construction de l'identité religieuse dans le contexte de la modernité : " les identités religieuses ne peuvent plus être considérées comme des identités héritées, même si l'on admet que l'héritage est toujours remanié. Les individus construisent leur propre identité socioreligieuse à partir des diverses ressources symboliques mises à leur disposition, et/ou auxquelles ils peuvent avoir accès en fonction des différentes expériences dans lesquelles ils sont impliqués. L'identité s'analyse comme le résultat, toujours précaire et susceptible d'être remis en question, d'une trajectoire d'identification qui se réalise dans la durée » (2001: 70).

Ce bricolage identitaire, qui part de l'individu, ne signifie pas pour autant qu'aucun adulte n'intervient. Andrea Riccardi rapporte que c'est un prêtreouvrier qui l'initie au monde des bidonvilles de la périphérie (1996: 10). Avec un autre prêtre, lui et son groupe découvrent la dimension liturgique de la foi chrétienne: "Tandis que se constituait une sorte de pré-Sant'Egidio, je découvrais l'aspect liturgique qui manquait à mon expérience » (ibid.: 10). Mais ces adultes n'ont aucunement le rôle de transmetteurs d'un bagage religieux qu'il faudrait accepter en bloc. Ils sont des éléments parmi d'autres de la trajectoire d'identification du groupe. Les deux ecclésiastiques ne peuvent d'ailleurs avoir de mainmise sur le groupe de par leur situation : le prêtre-ouvrier est qualifié de «très marginal » (id.) ; l'autre prêtre est « en difficulté » (id.)

L'exemple de Sant'Egidio atteste l'importance des ruptures de la mémoire ${ }^{4}$ dans le cadre de la modernité religieuse avancée et relativise la crise de transmission religieuse du XIX ${ }^{e}$ siècle. Le rapport de la communauté à son nom est de

4. "Les écarts repérables entre les univers culturels des différentes générations ne correspondent plus seulement aux ajustements que l'innovation et l'adaptation aux données nouvelles de la vie en société rendent nécessaires. Elles localisent de véritables fractures culturelles qui atteignent en profondeur les identités sociales, le rapport au monde et les capacités de communication des individus. Elles correspondent à un remaniement global des références collectives, à des ruptures de mémoire, à une réorganisation des valeurs qui mettent en question les fondements mêmes du lien social " (Hervieu-Léger, 2001 : 62-63). 
ce point de vue significatif. Au départ, le groupe formé par A. Riccardi ne porte pas de nom : "Nous nous désignions nous-mêmes comme La Communauté " (Riccardi, 1996 : 53). Ce n'est qu'en 1974 que le groupe décide de se « baptiser » : « Nous avons eu de longues discussions, jusqu'à une heure avancée de la nuit, et finalement nous avons opté pour Sant'Egidio (c'est-à-dire Saint-Gilles en français), du nom du lieu où nous étions installés » (id.) Contrairement aux étudiants parisiens de 1835, l’organisation n'est pas placée rapidement dans une filiation spirituelle. Quand un nom est donné à l'organisation, il correspond moins à un saint du patrimoine catholique, choisi pour son message, qu'à un lieu géographique. Saint Gilles, qui aurait vécu au $\mathrm{VI}^{\mathrm{e}}$ siècle, n'évoque rien aux premiers membres si ce n'est un toponyme. C'est dans un second temps que la communauté, par le recours à l'histoire ${ }^{5}$, essaye de se l'approprier. La légende selon laquelle saint Gilles aurait protégé une biche traquée par le roi wisigoth Wamba est relue comme un signe de la défense du pauvre (ibid.: 53). Cette divergence des trajectoires d'identification est corrélée avec des modes de communalisation différenciés.

\section{La construction du mode de communalisation}

Le mode de communalisation renvoie au type de liens créés entre les membres d'une organisation. Nous voudrions montrer que les premiers membres de la Société de Saint-Vincent-de-Paul procèdent à une communalisation limitée de leur vie religieuse, tandis que ceux de la communauté de Sant'Egidio opèrent une communalisation quasiment totale de la leur.

\section{Saint-Vincent-de-Paul : une communalisation limitée et ouverte}

Comme on peut le lire dans le règlement de 1835 , la conférence n'a pas vocation à intervenir dans tous les domaines de la vie chrétienne de ses membres. Elle se cantonne à celui qui concerne la pratique de la charité : "La Société de charité doit s'appliquer à acquérir et à pratiquer toutes les vertus; il en est pourtant quelques-unes qui conviennent davantage à ses membres pour l'accomplissement des fonctions charitables dont ils se chargent ; il faut mettre de ce nombre l'abnégation de soi-même, la prudence chrétienne, un amour efficace du prochain, le zèle du salut des âmes, la mansuétude du cœur et des paroles, et surtout l'esprit de fraternité. » (Société de Saint-Vincent-de-Paul, 1836 : 10). La communalisation

5. Les ruptures de la mémoire religieuse contribuent peut-être à expliquer l'importance de la discipline historique au sein de la communauté de Sant'Egidio. Cette inclination pour l'histoire est reconnue par Andrea Riccardi lui-même historien et universitaire : "Tous les membres de Sant'Egidio ne sont pas des historiens! Il y a beaucoup d'assistants sociaux, de médecins, d'enseignants et d'autres. Mais il y a un certain goût pour l'histoire. Disons moins d'idéologie et plus d'histoire. Là est le nœud." (Riccardi, 1996 : 35). 
est fonctionnellement limitée à la dimension éthique de la religion. Elle est aussi bornée dans le temps. L'adhésion à la Société de Saint-Vincent-de-Paul demande un investissement réel mais circonscrit: une réunion par semaine en plus de l'activité caritative. L'engagement dans une conférence n'est donc pas exclusif d'engagements complémentaires. En 1834, trois des fondateurs de la Société, Lamache, Lallier et Ozanam, s'investissent dans un projet de conférences de carême à Notre-Dame, totalement disjoint de leur appartenance à la Société (Société de Saint-Vincent-de-Paul, 1960 : 23). Ozanam s'engage dans l'œuvre de la Propagation de la foi, à partir de 1838, pendant une dizaine d'années tout en continuant à s'occuper activement de Saint-Vincent-de-Paul (Drevet, 2001 : 113-131).

\section{Sant'Egidio : une communalisation complète et exclusive}

Inversement, la communauté de Sant'Egidio met en place une communalisation assez complète de la vie religieuse de ses membres. La communauté n'est pas fonctionnellement limitée à un aspect de la vie religieuse. À travers la mission d'évangélisation, énoncée par l'article 2 des statuts de la communauté ${ }^{6}$, c'est toute la vie chrétienne des membres qui est concernée. Le terme même de «communauté » implique un degré de communalisation assez poussé. Si les membres de Sant'Egidio ne vivent pas sous le même toit, leur engagement au sein de la communauté est fort et généralement exclusif même si rien n'est fixé de manière rigide : "L'engagement concret dans le travail de la communauté - le travail social que nous menons ensemble - varie beaucoup selon les personnes. Il y a de grands dévouements. Il y a aussi la possibilité d'avoir une présence moins active, pas seulement par choix personnel mais à cause des conditions concrètes de sa propre vie » (Riccardi, 1996:39). Outre la participation à une activité sociale, les membres ont une prière commune quotidienne : "Nous avons commencé en 1973 à prier chaque soir. Cette pratique n’a jamais été interrompue jusqu'à aujourd'hui » (ibid. : 29). Du fait de cet investissement important, un certain nombre de membres sont célibataires (c'est le cas d'Andrea Riccardi) ou mariés sans enfants.

Cette communalisation intense est naturellement exclusive. À l'origine, les contacts des membres avec les autres organisations religieuses, ou tout simplement avec les autres chrétiens, étaient limités. Riccardi, plus de vingt-cinq ans après la fondation, confesse : "Je pense que le grand risque que courent les expériences communautaires, c'est le sectarisme qui conduit à vivre enfermé sur soi-même et à se prendre pour le Messie ou à se croire investi de l'Évangile... »

6. «La fin première de la communauté de Sant'Egidio est l'évangélisation "mission essentielle de l'Église" (Evangeli Nuntiandi, 14). La communauté est consciente que la parole de Jésus “je dois annoncer la bonne nouvelle du royaume de Dieu” (Lc, 4,13) s'applique aussi à elle» (Riccardi, 1996, p. 40). 
(ibid. : 20). Cet écueil du sectarisme n'a pas été évité par la communauté qui, selon les propres mots de son fondateur, est à l'origine un groupe "chaud » et "fermé " ${ }^{7}$. Ce n'est que vers le milieu des années soixante-dix que des liens avec le reste du monde catholique se développent. En 1973, la communauté décide de rendre publique ses prières (ibid.: 30). En 1974, des contacts sont tissés avec des chrétiens de Rome et des organisations caritatives dans le cadre de la participation d'un congrès organisé par le cardinal Poletti sur « Les attentes en charité et en justice du diocèse de Rome » (ibid. : 52). Première confrontation avec les autres communautés, l'événement est considéré par A. Riccardi comme " une expérience ecclésiale importante » $(i d$.

\section{Mode de communalisation et dimension protestataire}

Il semble que le caractère «protestataire » de la fondation de Sant'Egidio peut expliquer le caractère globalisant de la communalisation mise en œuvre. La communauté de Sant'Egidio naît d'une volonté de "construire une manière nouvelle de vivre l'Église" (ibid.: 19), de «changer l'homme " (ibid.: 8). Elle est générée par une indignation contre la pauvreté des bidonvilles et l'égoïsme bourgeois ${ }^{8}$. Elle prend aussi sa source dans la conversion personnelle d'Andrea Riccardi, suite à la lecture de l'Évangile, vu comme une force de changement. Or, il existe des affinités entre la conversion protestataire et l'entrée dans une communauté relativement fermée : "Cette dimension protestataire de la conversion nourrit l'aspiration utopique à l'entrée, symbolique et effective, dans une communauté idéale opposable à la société environnante. Cette espérance peut s'actualiser, aujourd'hui comme à d'autres époques, dans des créations communautaires. Celles-ci s'efforcent d'anticiper, à l'échelle réduite du groupe des convertis, un style de relations sociales et interpersonnelles opposables à la société environnante. Les nouveaux groupes religieux que J. Beckford décrit comme des "groupes refuges", offrant à leurs membres la protection d'une communauté et d'un mode de vie entièrement intégré, sont une illustration contemporaine de cette dynamique de “l'utopie pratiquée » (Hervieu-Léger,

7. A. Riccardi raconte la manière «fermée " dont se passaient les prières jusqu'en 1974 : « Pour nous, prier, jusqu'en 1974, c'était se réunir dans une pièce, ouvrir la Bible, lire les prières, toujours dans un milieu clos. " (ibid.: 30 ).

8. « (...) la deuxième étape fut la connaissance du monde en dehors de Rome : les borgate, le monde de la banlieue pauvre, les bidonvilles de la périphérie. (...). À ce stade de la démarche revenait le discours sur les ouvriers. Dans le monde étudiant on disait : "Étudiants et ouvrier unis dans la lutte!" Moi je répondais que l’Évangile nous conduit à découvrir cette Rome que nous ne connaissions pas. Pourquoi ? Parce que nous étions des bourgeois ; cette Rome que nous ne connaissions pas, c'était la Rome de la périphérie, celle d'où venaient nos femmes de ménage. Alors allons découvrir les baraques! C'était pour un jeune bourgeois une expérience traumatisante. Découvrir cette pauvreté, ces gens qui vivaient dans des bidonvilles, c'était découvrir que le tiers-monde était à Rome. La tromperie de la ville bourgeoise consiste à ne pas montrer les pauvres» (ibid.: 9). 
2001 : 140-141). Le caractère protestataire et, partant, utopique de la fondation de la Société de Saint-Vincent-de-Paul est quant à lui beaucoup moins prononcé. Le moteur initial de la fondation, c'est la volonté de montrer, face aux attaques dont l'Église est l'objet, la vitalité du catholicisme par les œuvres ${ }^{9}$. La volonté de lutter contre la pauvreté et l'injustice n'est que seconde. Le caractère plus protestataire de la fondation de la communauté de Sant'Egidio est donc un élément d'explication du caractère global de sa communalisation.

\section{Des régimes de validation du croire différenciés}

Si l'on fait du type de validation du croire le principe des formes de communalisation religieuse (Hervieu-Léger, 2001 : 190-196), nous pouvons avoir une autre piste d'explication intéressante. La Société de Saint-Vincent-de-Paul privilégie une validation institutionnelle du croire. Cette validation institutionnelle se fait en interne pour toutes les questions ayant trait aux croyances concernant l'organisation de la Société ou les pratiques qui doivent y être mises en œuvre. C'est un président institué qui se voit confier la tâche de définir l'orthodoxie et l'orthopraxie. On notera que le premier président élu, Bailly, est l'homme mûr du groupe : il a quarante-deux ans en 1833. Sa différence d'âge avec les étudiants semble le mettre naturellement dans une position d'arbitre et de référent. Concernant la croyance en la légitimité de la Société de Saint-Vincent-de-Paul, et plus généralement pour tout ce qui concerne les dogmes et la foi catholiques, les fondateurs ont, dès le début, fait appel à une validation institutionnelle externe du croire auprès de la hiérarchie ecclésiastique. Ainsi, en avril 1833, Emmanuel Bailly, vers qui se sont tournés les étudiants, les envoie " consulter le curé de la paroisse de Saint-Étienne-du-Mont » (Société de Saint-Vincent-de-Paul, 1960 : 15) sur le territoire duquel se situe l'activité qu'ils veulent mettre en place. Par la suite, les confrères prennent soin d'inviter des représentants de l'Église institutionnelle pour légitimer l'existence de leur organisation. Dès le 27 juin 1834, soit à peine un an après la fondation, le curé de Saint-Étienne du Mont est convié à assister à une réunion durant laquelle on lui lit un rapport sur les activités de la conférence ${ }^{10}$. En avril 1838, ce sont des représentants de l'archevêque de Paris qui sont présents lors de l'Assemblée générale (Société de Saint-Vincent-de-Paul, 1836 : 5). Ce choix de validation institutionnelle du croire apparaît bien dans ce passage $\mathrm{du}$

9. Selon le Récit des origines, les mots par lesquels Ozanam persuada ses amis de fonder la première conférence de charité sont les suivants : "Combien il est malheureux, (...), de voir le catholicisme, de voir notre sainte mère l'Église ainsi attaquée, travestie, calomniée. Restons sur la brèche pour faire face aux attaques. Mais n'éprouvez-vous pas comme moi, le désir, le besoin d'avoir, en dehors de cette conférence militante, une autre réunion composée exclusivement d'amis chrétiens, et toute consacrée à la charité ? Ne vous semble-t-il pas qu'il est temps de joindre l'action à la parole, et d'affirmer par des œuvres la vitalité de notre foi ? " (Société de Saint-Vincent-de-Paul, $1960:$ 14-15).

10. Ce rapport a pour auteur Gustave Colas de La Noue. Il a été retrouvé tardivement et reproduit à l'occasion du procès de béatification d'Ozanam (Sacra Congregatio pro causis sanctorum officium historicum, $1980: 348-349$ ). 
Règlement: "Nous nous souviendrons sans cesse que nous ne sommes que des laïcs et, pour la plupart, des jeunes gens, sans mission pour enseigner les autres. Nous aurons donc, sous ce rapport et tous les autres, la plus grande déférence pour les conseils qui nous seraient donnés par la Société et par ses chefs : nous suivrons surtout avec une docilité absolue la direction que les supérieurs ecclésiastiques peuvent juger à propos de nous donner» (ibid. : 49-50).

Il semble que la validation du croire diffère au sein de la communauté de Sant'Egidio naissante. Cette organisation a mis en place un système de validation communautaire du croire que l'on peut définir ainsi : " En régime de validation communautaire du croire, c'est le groupe comme tel qui constitue l'instance de légitimation. Dans ce cas, la cohérence des comportements de chacun des membres au regard des normes, des objectifs et plus largement du rapport au monde définis par le groupe constitue le critère principal de la vérité du croire partagé. L'égalitarisme de principe, supposé régir les relations au sein du groupe, ne signifie pas qu'aucun leader ne puisse émerger : mais celui-ci est toujours supposé s'exprimer au nom du groupe tout entier ; il est la voix du groupe » (Hervieu-Léger, 2001 : 186). Dans la communauté de Sant'Egidio naissante, il n’y a pas, comme dans la conférence de charité, d'adulte référent qui puisse orienter les décisions : "Nous étions seuls et responsables de nous-mêmes. Il n'y avait pas de prêtre parmi nous " (Riccardi, 1996:18). C'est l'Évangile qui est la seule autorité et le seul guide. L'autorité de l'Église n'est pas prise en compte: "Quant à moi, je pensais qu'il fallait changer l'homme. C'est dans ce sens que je lisais l'Évangile (...). Mais je le lisais en me tenant à distance de l'Église. Pour moi, l'Évangile, c'était ce livre ancien, le livre de la foi, la parole de Dieu, quelque chose qui permettrait de changer l'homme » (ibid. : 8). Le régime communautaire de validation du croire apparaît bien dans la cooptation des nouveaux membres : "Si je veux être membre de Sant'Egidio, je me reconnais dans une certaine spiritualité, et je suis accepté par les autres comme tel. L'adhésion est le fruit de deux volontés qui se rencontrent : tu le veux et nous le voulons. Celui qui veut venir fait une démarche vers la communauté, et celle-ci l'accepte. Il y a bien l'expression d'une double volonté » (ibid. : 42). Ce n'est pas un règlement ou une personnalité élue qui décide de l'admission d'une personne dans la communauté, c'est la communauté dans son ensemble. L'absence de validation institutionnelle interne du croire s'accompagne d'une absence de validation externe de la légitimité des buts et de l'action de la communauté auprès de la hiérarchie catholique, tout du moins dans les premières années de la fondation. En effet, au départ, le groupe a tendance à vivre «en autarcie » par rapport à l'institution religieuse. Il ne semble pas y avoir de désir ou de souhait de reconnaissance. Sant'Egidio choisit de se situer volontairement dans une situation marginale tant au niveau institutionnel que spatial ${ }^{11}$ : «Mais nous comme groupe, nous étions un peu en marge.

11. L'installation de la communauté en périphérie est une traduction spatiale du choix de la marginalité institutionnelle (Riccardi, $1996: 14$ ). 
Nous allions dans la banlieue de Rome, dans un quartier assez pauvre, qui s'appelait Primavalle, où nous avions notre siège dans le sous-sol. Je me souviens de ce que disait le curé : "dans ma paroisse, je les ai tous, les extrémistes de droite, les extrémistes de gauche, les extrémistes d'Église”. Nous étions les extrémistes d'Église, ou comme il disait les "extra-ecclésiaux", comme il y a les "extraparlementaires " (ibid.: 11).

Ce n'est que dans un second temps que s'opère un basculement progressif vers un régime de validation institutionnelle au moins pour ce qui concerne la légitimité des buts et de l'action de la communauté. Après la participation du groupe au Congrès sur «Les attentes en charité et en justice du diocèse de Rome ", le cardinal Poletti noue des liens avec ses responsables. La validation institutionnelle de l'existence de la communauté au niveau local est donc en cours. La validation au niveau de l'Église universelle ne s'effectue qu'après l'élection de Jean-Paul II au pontificat de manière relativement fortuite. Karol Wojtyla s'était mis à visiter les quartiers en tant qu'évêque de Rome. Alors qu'il passait devant les fenêtres de la crèche créée par la communauté, A. Riccardi l'interpelle et lui propose de rencontrer son groupe (ibid.: 54). Des contacts se nouent et petit à petit la situation de la communauté au sein de l'Église est "régularisée " (id.). Sant'Egidio évolue donc vers une validation institutionnelle externe mais tardivement, et presque malgré elle. À la différence de la conférence de SaintVincent-de-Paul, ce n'est pas l'organisation qui se présente à l'institution ecclésiale, c'est l'institution ecclésiale qui vient à la rencontre de l'organisation.

En quoi ces régimes différenciés de validation du croire peuvent-ils expliquer les différences de communalisation ? Nous l'avons vu, la Société de Saint-Vincentde-Paul n'a pas pour fonction de valider toutes les croyances religieuses de ses membres. Elle se décharge de ce rôle sur l'institution ecclésiale et sur un président élu. Par conséquent l'investissement religieux dans la structure n'a pas besoin d'être total. La communauté de rattachement première est l'Église universelle qui, en dernier ressort, détient l'autorité : "En régime de validation institutionnelle, c'est l'ensemble des croyants passés, présents et futurs qui constituent la "communauté" authentique. Les petites communautés sont des condensations historiques de la lignée croyante. Elles n'épuisent pas la réalité de la "grande communauté” (le Peuple élu, l’Umma, l’Église) qui est leur référence. L'autorité religieuse institutionnelle est celle à qui est reconnue le droit de parler légitimement au nom de la "grande communauté " (Hervieu-Léger, 2001 : 199). Cela explique que des membres des conférences puissent également être investis dans d'autres œuvres religieuses. En revanche, la communauté de Sant'Egidio naissante est, pour ses membres, la seule instance de validation des croyances religieuses. Ceux-ci y investissent donc la totalité de la dimension religieuse.

La dynamique de communautarisation révélée par le "cas Sant'Egidio » s'accompagne, semble-t-il, d'une tendance à la revalorisation du charisme. 


\section{La durée de la période de domination charismatique}

Selon Weber, le charisme est « une qualité extraordinaire d'un personnage qui est, pour ainsi dire, doué de forces ou de caractères surnaturels ou surhumains ou tout au moins en dehors de la vie quotidienne, inaccessibles au commun des mortels ». Le détenteur du charisme est " considéré comme envoyé de Dieu ou comme un exemple, et en conséquence considéré comme un chef » (Weber, 1971 : 249). Autour de lui se forme un groupe de disciples que Weber appelle "communauté émotionnelle "(Ouedraogo, 1993 : 142). L'organisation de la communauté émotionnelle par la domination charismatique est temporaire car le porteur de charisme se trouve forcément confronté à l'échec un jour ou l'autre. D'où la nécessité d'une transition vers une nouvelle forme de domination plus institutionnelle. Ce passage de la domination charismatique à la domination institutionnelle diffère pour la Société de Saint-Vincent-de-Paul et la communauté Sant'Egidio.

\section{Saint-Vincent-de-Paul : brève domination charismatique}

Dans le cas des origines de la Société de Saint-Vincent-de-Paul, le principal porteur de charisme est Frédéric Ozanam, étudiant en droit âgé de vingt ans. Les récits de fondation mettent l'accent sur ses dons extraordinaires, sur son rayonnement et son pouvoir de persuasion. Ainsi, Amand Chaurand, un des premiers membres de la Société, le décrit-il comme le "guide et le soutien » des étudiants de Paris qui se regroupaient, selon lui, "sous l'égide de sa fermeté et le patronage de sa parole » (1856). Selon le "Récit des origines », il est l'élu par lequel Dieu fait entendre sa révélation aux futurs premiers confrères : "Quand le petit groupe se sépara, chaque membre emportait au cœur le trait enflammé que Notre Seigneur Jésus-Christ venait d'y faire entrer par la parole du jeune étudiant [Ozanam]" (ibid. : 15).

Ce qui est remarquable, c'est que cette domination charismatique est de très brève durée. Presque immédiatement, la communauté émotionnelle qui s'est formée autour d'Ozanam passe à un fonctionnement institutionnel par l'élaboration de règles dès la première réunion (Société de Saint-Vincent-de-Paul, 1960 : 18). Très vite, les normes, discutées en commun, deviennent très précises, notamment pour le déroulement de la réunion : "À l'ouverture de chaque séance, le Président prononcera la prière Veni Sancte Spiritus, suivie de l'oraison et d'une invocation à saint Vincent de Paul. On fait ensuite une lecture de piété, dans un livre choisi par le Président. Chacun est appelé à le faire à son tour (...). Le secrétaire donne lecture du procès-verbal de la séance précédente. Chaque membre est admis à faire des observations sur ce procès-verbal. S'il y a lieu, le Président proclame l'admission des candidats présentés à la séance précédente, et invite ceux qui les ont présentés à leur annoncer leur admission (...). » (Société de Saint-Vincentde-Paul, 1836 : 31-32). Cette routinisation permet à Ozanam de s'effacer derrière Emmanuel Bailly, qui accède à la présidence dès la première réunion (id.), bien 
que sa relation avec les étudiants soit tout sauf charismatique comme l'atteste ce témoignage de l'un des premiers membres : "M. Bailly a été à cette époque un réfrigérant, utile sans doute, mais il n'avait pas le don de plaire à tous (...) M. Bailly était le représentant des sentiments surannés et l'homme des mesures habiles ou supposées telles ${ }^{12}$. " Ozanam exerce quant à lui une responsabilité institutionnelle seconde : en 1835, il est nommé « vice-président pour la première section » (Société de Saint-Vincent-de-Paul, 1960 : 33). L'organisation repose donc très vite sur l'obéissance à un pouvoir institutionnalisé, qui se conforme à des normes établies rationnellement et codifiées dans un règlement en décembre 1835 .

\section{Sant'Egidio : domination charismatique plus étendue}

Dans le cas de la communauté de Sant'Egidio, le principal dépositaire du charisme est Andrea Riccardi. C'est lui qui, par son itinéraire de foi, par sa conversion, par ses lectures théologiques, suscite l'adhésion chez ses camarades de lycée. Touché conjointement par le bouillonnement de la seconde moitié des années soixante et par la découverte de l'Évangile, il se sent poussé à fonder une communauté émotionnelle : "Pour ma part, je pensais à 68 et je subissais l'attraction de l'Évangile (...) L'Église me paraissait un peu lointaine. La paroisse ne me plaisait pas beaucoup ni l'Action catholique. Je ressentais le besoin de faire quelque chose dans mon lycée. Je mis alors sur pied un premier groupe de lycéens qui se réunit en février 1968 »(Riccardi, 1996 : 9).

Contrairement à la Société de Saint-Vincent-de-Paul, il n'y a pas immédiatement de développement d'une autorité institutionnelle. Les étudiants se veulent "seuls et responsables [d'eux]-mêmes » (ibid.: 18). Les règles écrites sont longtemps inexistantes. La communauté se pense à ses débuts davantage comme famille que comme structure ce qui laisse le champ libre à l'exercice de la domination charismatique. Une évolution se dessine néanmoins au milieu des années quatre-vingts par l'adoption de statuts. La communauté se constitue en association publique de laïcs. Un conseil et un président sont élus tous les quatre ans (ibid.: 40). Andrea Riccardi transforme ainsi sa domination charismatique en domination bureaucratique en se faisant élire président. La communauté fait le choix, contrairement à la Société de Saint-Vincent-de-Paul, de prolonger la domination du détenteur du charisme sous une forme institutionnalisée.

\section{Éléments d'explication}

La longueur de la période de domination charismatique, et sa perpétuation sous une forme institutionnalisée, dans la communauté de Sant'Egidio peut illustrer la désinstitutionalisation et l'individualisation du croire : "l'affaissement

12. Lettre de Paul Brac de La Perrière à Amélie Ozanam, Lyon, le 6 juin 1889, Archives Laporte-Ozanam, transcription Raphaëlle Chevalier-Montariol. 
des régimes institutionnels de la validation du croire et le besoin croissant de confirmation mutuelle et communautaire des "petites vérités" produites par les individus favorisent considérablement le rôle de personnalités qui peuvent témoigner par leur expérience personnelle, d'une antériorité sur le chemin de la vérité et donc d'une capacité d'initier ceux qui sont disposés à les suivre » (HervieuLéger, 2001 : 188). Dans un contexte où la confiance dans la légitimité normative de l'institution se délite, l'importance d'individus apparaissant comme dotés de dons extraordinaires augmente. Peuvent aussi rendre compte de l'importance grandissante des personnalités charismatiques « la spectacularisation généralisée de la vie sociale qui correspond à l'avènement de la civilisation des médias » ainsi que la culture de la performance « qui valorise tous ceux, quel que soit le domaine dans lequel ils déploient leurs activités, qui sont parvenus à sortir des sentiers battus, à s'arracher aux routines de la vie ordinaire et à “aller jusqu'au bout d'eux-mêmes» (id.) Dans le cas d'Andrea Riccardi, cette explication marche pleinement. Cet universitaire qui consacre sa vie aux pauvres et à la paix dans le monde a quelque chose d'extraordinaire et de fascinant.

\section{Conclusion}

À la question de Dominique Chivot "votre expérience était-elle vraiment originale à l'époque ? ", Andrea Riccardi répond : " Nous avions en effet l'impression de vivre quelque chose d'original. Mais avec les années et la maturité, un constat s'impose : quand on s'engage dans une aventure originale, on revit de manière responsable une expérience que d'autres ont vécu en d'autres lieux et saisons » (Riccardi, 2001 : 21). Peut-être l'expérience de Sant'Egidio reproduitelle d'autres expériences qui lui sont contemporaines. Mais elle s'est avérée bien différente de celle vécue par les premiers confrères de Saint-Vincent-de-Paul entre 1833 et 1835 . Le contexte dans lequel se déroule la fondation n'est pas le même et ne permet pas que la même expérience se produise une seconde fois. Entre 1833 et 1968, la croyance en l'autorité de l'institution s'est délitée, la mémoire collective religieuse s'est fracturée. La mise en perspective de la fondation de la Société de Saint-Vincent-de-Paul et de la communauté de Sant'Egidio nous fait ainsi saisir la réalité concrète de la modernité religieuse avancée.

Malgré ces différences, on peut noter une tendance à l'évolution progressive de la communauté de Sant'Egidio vers la tradition. Les efforts de recherche historique cherchent à retisser le lien avec les générations croyantes précédentes. Le rapprochement vers l'institution provoque un réaménagement du régime de validation du croire et des modes de communalisation. Le charisme tend à se routiniser et à s'inscrire dans une perspective institutionnelle : éléments qui montrent une certaine capacité d'adaptation de l'institution.

Charles MERCIER

Université Paris IV - IUFM charles.mercier@paris.iufm.fr 


\section{Bibliographie}

Barbiche Bernard, Franconnet Christine, 2006, Frédéric Ozanam (1813-1853), Un universitaire chrétien face à la modernité, Paris, Éditions du Cerf.

BerTHIER De SAUVIGny Guillaume de, 1948, Un type d'ultra-royaliste : le comte Ferdinand de Bertier (1782-1864) et l'énigme de la Congrégation, thèse de doctorat, Faculté des lettres de l'Université de Paris.

BRÉMARD Magdeleine, 1967, Mentalité sociale, intellectuelle, religieuse des étudiants de Paris au lendemain de 1830, Paris, Diplôme d'Études Supérieures d'Histoire, Université de la Sorbonne.

Chaurand Amand, 1856, La Gazette de Lyon, 18 février.

Cholvy Gérard, 2000, "Les organisations de jeunesse entrent dans l'histoire ", Revue de l'histoire de l'Église de France, t. 86, 27, pp. 347-361.

-, 2003, Frédéric Ozanam, L'engagement d'un intellectuel catholique an XIX ${ }^{e}$ siècle, Paris, Fayard.

Drevet Richard, 2001, "Frédéric Ozanam et la Propagation de la foi à Lyon : les raisons d'un échec ", in Faculté de théologie de Lyon, Frédéric Ozanam, Paris, Bayard, pp. 113-131.

Goyau Georges, 1926, «Frédéric Ozanam et la fondation des Conférences de SaintVincent-de-Paul ", La Documentation catholique, 325.

Hervieu-Léger Danièle, 2001, Le Pèlerin et le converti, la religion en mouvement, Paris, Flammarion, coll. "Champs".

-, 1993, La Religion pour mémoire, Paris, Éditions du Cerf.

Mercier Charles, 2006, La Société de Saint-Vincent-de-Paul, une mémoire des origines en mouvement, 1833-1914, Paris, L'Harmattan.

Montlosier François Dominique de Reynaud de, 1826, Mémoire à consulter sur un système religieux et politique tendant à renverser la religion, la société et le trône, Paris, A. Dupont \& Roret.

OuEDRAOGO Jean-Martin, 1993, "La réception de la sociologie du charisme de M. Weber », Archives de Sciences sociales des religions, 83, pp. 141-157.

RicCARDI Andrea, 1996, Sant'Egidio, Rome et le monde. Entretiens avec Jean-Dominique Durand et Régis Ladous, Paris, Beauchesne.

-, 2001, Sant'Egidio, l'Évangile au-delà des frontières. Entretiens avec Dominique Chivot, Paris, Bayard.

Sacra Congregatio pro causis sanctorum officium historicum, 1980, Massilien. Seu Parisien. Beatificationis et canonizationis servi Dei Fridirici Ozanam patris familias, primarii fundatoris Societatis conferentiarum S. Vincentii a Paulo disquisitio de vita et actuasitate, Rome.

Société de Saint-Vincent-de-Paul, 1836, Règlement, Paris, Imprimerie E. J. Bailly \& Cie. -, 1960 (s.d.), "Origines de la Société de Saint-Vincent-de-Paul », in Origines et fondateurs de la Société de Saint-Vincent-de-Paul, Paris, Société de Saint-Vincent-de-Paul.

-, 2003, Promouvoir la Société de Saint-Vincent-de-Paul, Paris, Société de Saint-Vincentde-Paul.

Sullivan Louise, 2007, Sœur Rosalie Rendu. Une passion pour les pauvres (Sister Rosalie Rendu: A Daughter of Charity On Fire with Love for the Poor, 2006), Paris, Médiaspaul. Weber Max, 1971, Économie et société, Paris, Plon.

XXX, 1926, "Quelques observations ", La Documentation catholique, 325. 


\section{Résumé}

Cet article cherche à comparer les fondations de deux organisations catholiques : la Société de Saint-Vincent-de-Paul et la communauté Sant'Egidio. La première est créée à Paris entre 1833 et 1835, la seconde se construit à Rome entre 1968 et 1986. Bien que le but (le service des pauvres) et les membres (les étudiants catholiques) des deux organisations soient similaires, les différences entre les deux expériences sont importantes. La construction de l'identité religieuse, le type de liens instaurés entre les membres et la place réservée au charisme révèlent que, entre 1833 et 1968, la croyance en l'autorité de l'institution catholique s'est délitée et que la mémoire religieuse s'est fracturée. La mise en perspective de la fondation de la Société de Saint-Vincent-de-Paul et de la Communauté de Sant'Egidio illustre le phénomène de la modernité religieuse avancée.

Mots-clés : identité, transmission religieuse, charisme, fondation, communauté.

\section{Abstract}

This article aims at comparing the foundation of two Catholic organizations: the Society of Saint Vincent de Paul and the Community of Sant'Egidio. The first one is created in Paris between 1833 and 1835. The second one is born in Rome between 1968 and 1986. Althought the purpose (the service to poor people) and the members (Catholic students) of both organizations are similar, the differences between both experiences are important. The building of the religious identity, the kind of links established between the members, the place dedicated to charism show that, between 1833 and 1968, the belief in the authority of the Catholic institution has desintegrated and that the religious memory has split. The comparison between the foundations of the Society of Saint Vincent de Paul and the Community of Sant'Edigio exemplifies the phenomenon of advanced religious modernity.

Key words: identity, religious transmission, charism, foundation, community.

\section{Resumen}

Este artículo busca comparar las fundaciones de dos organizaciones católicas, la Sociedad San Vicente de Paul y la comunidad San Egidio. La primera es creada en París entre 1833 y 1835, la segunda ve la luz en Roma entre 1968 y 1986. Aunque el origen (el servicio de los pobres), y los miembros (los estudiantes católicos) de las dos organizaciones son similares, las diferencias entre las dos experiencias son importantes. La construcción de la identidad religiosa, el tipo de lazos instaurados entre los miembros y el lugar reservado al carisma revelan que, entre 1833 y 1968, la creencia en la autoridad de la institución católica se ha desmoronado y la memoria religiosa se ha fracturado. La puesta en perspectiva de la fundación de la Sociedad San Vicente de Paul y de la Comunidad San Egidio ilustra el fenómeno de la modernidad religiosa avanzada.

Palabras clave: identidad, transmisión religiosa, carisma, fundación, comunidad. 